\title{
Common conditions of abutment teeth and their severity in subjects requesting fixed dental prostheses at Watim Dental Hospital, Rawalpindi.
}

\footnotetext{
1. FCPS

Assistant Professor Prosthodontics Multan Medical and Dental College, Multan.

2. FCPS

Assistant Professor Prosthodontics Watim Dental College, Rawalpindi.

3. FCPS

Assistant Professor Operative Dentistry

Watim Dental College, Rawalpindi.

4. FCPS

Consultant Prosthodontics

Polyclinic Hospital Islamabad.

5. FCPS

Assistant Professor Prosthodontics Foundation University College of Dentistry, Islamabad.

6. FCPS

Assistant Professor Oral and Maxillofacial Surgery Shifa College of Dentistry Rawalpindi.

Correspondence Address: Dr. Zarah Afreen

Department of Prosthodontics

Watim Dental College, Rawalpindi. zarahsufian@yahoo.com
}

Article received on:

26/10/2020

Accepted for publication:

07/12/2020

\begin{abstract}
Aamna Khalil', Zarah Afreen², Ammarah Afreen ${ }^{3}$, Umer Javed ${ }^{4}$, Shoaib Rahim ${ }^{5}$, Adil Asim ${ }^{6}$
\end{abstract}
ABSTRACT... Objective: The objective of this study was to examine the abutments in subjects requesting for fixed dental prosthesis (FDP) and to determine the frequency of compromised coronal conditions and their severity levels. Study Design: Descriptive, Cross-sectional Study. Setting: Department of Prosthodontics at Watim Dental Hospital, Rawalpindi. Period: August 2019 to March 2020. Material \& Methods: Conducted on 163 subjects. Abutments were evaluated in terms of clinical crown length, crown inclination, caries and/or fracture, type and quality of restoration. Abutments were categorized on the basis of complexity of the procedure required to make the abutment usable for fixed dental prosthesis. Arch-wise distribution of the abutment with compromised conditions and their proportion was also calculated. Results: The mean age of the participants was $39.42+11.56$ years with male to female ratio of with 2.2:1. Maximum number of the patients had edentulous spaces located in the mandibular posterior region. Tooth no. 46 displayed the highest frequency of use as abutment while tooth no. 18, $28,31,32,42$ displayed the least frequency of use as FDP abutment. About half of the abutments $(47 \%)$ had compromised restoration quality. On the basis of the degree of compromise, $41.1 \%$ of the abutments were usable without further treatment or required a simple corrective procedure, $45.4 \%$ required a relatively complex procedure to make them useable while $11 \%$ had sufficient compromise to preclude their use for FPD support. Only $2.5 \%$ of abutments were classified as adequate. Conclusion: About half of the studied abutments in subjects reporting for replacement of missing teeth with FDP were found to be compromised and a substantial proportion required complex restorative treatment/re-treatment prior to be used as FDP abutments.

Key words: Abutments, Compromised Coronal Conditions, Fixed Dental Prosthesis, Severity Levels.

Article Citation: Khalil A, Afreen Z, Afreen A, Javed U, Rahim S, Asim A. Common Conditions of Abutment Teeth and Their Severity in Subjects Requesting Fixed Dental Prostheses at Watim Dental Hospital, Rawalpindi. Professional Med J 2021; 28(7):253-259. https://doi.org/10.29309/TPMJ/2021.28.02.6182

\section{INTRODUCTION}

Different types of fixed prostheses are available to replace missing teeth. ${ }^{1}$ Fixed dental prosthesis (FDPs) have worldwide popularity despite of introduction of implants and implant supported fixed prosthesis..$^{2-3}$ As FDPs provide greater retention and stability in addition to comfort, it is more or less considered as next best treatment option to implants. ${ }^{4}$ When replacing missing teeth, conventional FDPs have long been a treatment option. Both cantilever and fixed-fixed conventional fixed partial dentures show high survival rates. ${ }^{5}$ Longevity and complication rate of FDPs is highly influenced by the skill level of the dentist and his or her academic knowledge. The placement of FDPs is one of the most common procedures done in routine practice. ${ }^{4}$

If properly planned and correctly designed, FDPs not only provide predictable function but also enhance the aesthetics and prove to be good value for money to the patient. On the other hand, a poorly designed prosthesis is likely to fail prematurely and leads to irreversible damage to the teeth and supporting structures. ${ }^{4}$ There is no doubt that success of FDPs depends on various factors such as design, material used and fabrication technique, but condition of natural abutments also plays vital role. ${ }^{2}$ 
Quite often natural teeth used as abutments for FDPs are usually not virgin and ideally positioned in the dental arches. Therefore, the principles of tooth preparations are not applicable to such teeth. The natural tooth as abutment must exhibit specific features that could indicate the possibility of its use for the FDP. After preparation, the abutment tooth should effectively facilitate support and retention which is important for the long term success of FDP. ${ }^{2}$ In this regard several features that could positively or negatively influence the FDP treatment outcome have been proposed. ${ }^{2-3}$ Some of the features rendering an abutment compromised may be correctable prior to the tooth preparation with procedures ranging from simple to complex ones while still others when exhibited may exclude the use of the abutment in the FDP support. To start with, the crown of the abutment tooth must have sufficient occlusocervical (OC) length and other features so that upon preparation it exhibits resistance to vertical and horizontal dislodgement forces exerted on the FDP during function. ${ }^{3}$ Abnormally inclined abutments are encountered quite frequently either towards or away from the edentulous space. According to a study, prevalence of mesioangular inclination was $50 \% .^{5}$ The prevalence of caries is from as low as $12.9 \%$ to as high as $59.1 \%{ }^{6,7}$ Acceptable restorative conditions after endodontic therapy of FDP abutments have been shown to provide favorable therapeutic outcome related to FDP survival. ${ }^{8,9}$ The type of restorative material also plays important role. Bernardo et al found that composite restorations had annual failure rates of $9.43 \% .{ }^{10}$ According to a study on quality of dental restorations, the percentage of unacceptable restorations increased by $28 \%$ and $17 \%$ with regard to marginal integrity and anatomic form respectively. ${ }^{11}$

Clinical evaluation of an abutment tooth for use in FDP is of paramount importance. The present study therefore aimed to investigate the compromised conditions and their severity levels to determine whether to use the abutment for FDP support or not. This will provide an idea on the conditions that need to be given special attention while examining the abutments.

\section{MATERIAL \& METHODS}

A descriptive cross sectional study was conducted in the department of Prosthodontics at Watim Dental Hospital, Rawalpindi. The study duration was six (06) months from August 2019 to March 2020 consisting sample size of 163 subjects. The sampling technique was non probability consecutive sampling technique. Both male and female patients having bounded saddle(s) in any segment of the maxillary and mandibular arch were included in this study. Exclusion criteria consisted of patients with deciduous teeth and advanced periodontal and periapical infections. Approval from hospital's ethical committee (Ref. no. WDC/2019/1099) was obtained. Subjects referred from OPD, fulfilling the inclusion criteria were invited to participate in the study and informed written consent was obtained regarding their willingness for participation in the study. Intra-oral examination was performed to evaluate abutment in terms of clinical crown length, crown inclination, caries and/or fracture, type of restoration and quality of restoration. Once detected, the compromised coronal condition was categorized on the basis of severity into category 1 (if the abutment can be used with or without the need for simple corrective procedures), category 2 (if relatively complex procedures are required for making the abutment useable) and category 3 (if the abutment could not be used for the support of the FDP). Details of the conditions for each and their levels are given in Annex 1. Data was entered in a structured data collection performa. Data relating to the compromised conditions was seen in all abutments including their levels and was recorded for each such abutment. Exclusion criteria were strictly followed to control confounders, effect modifiers and bias in the study results

The data were subjected to descriptive and analytical statistics using the software statistical package for social sciences (SPSS) version 17. Meanage, standard deviation (SD) and proportions of males and females were calculated. Arch-wise distribution of the abutments with compromised conditions, number of edentulous areas and distribution of teeth to be used as abutments were also computed. The proportion of the 
various abutments having compromised coronal conditions as per their number (notation) in the arch is calculated. The distribution of abutments and their levels of the mentioned conditions (morphology, integrity and restorative status) which renders them compromised is computed and tabulated. Proportion of abutment(s) offering the mentioned category of complexity for there to use in the FDP support is determined.

\section{RESULTS}

A total of 163 subjects were included in this study. The mean age of the participants was $39.42 \pm 11.56$ years with minimum age being 18 years and maximum being 62 years. Out of 163 participants, $112(68.7 \%)$ were males and 51 (31.3\%) were females. Maximum number of the patients had edentulous spaces located in the mandibular posterior region (Figure-1). Tooth no. 46 displayed the highest frequency of use as abutment while tooth no. 18, 28,31,32,42 displayed the least frequency of use as FDP abutment.

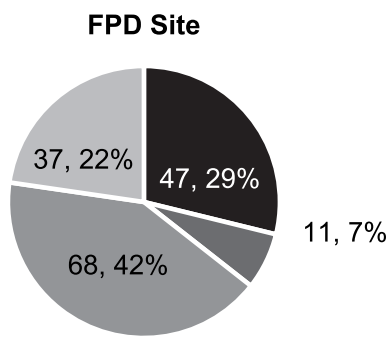

Maxillary Posterior Maxillary Anterior

Mandibular Posterior Mandibular Anterior

Figure-1. Distribution of the FPD sites.

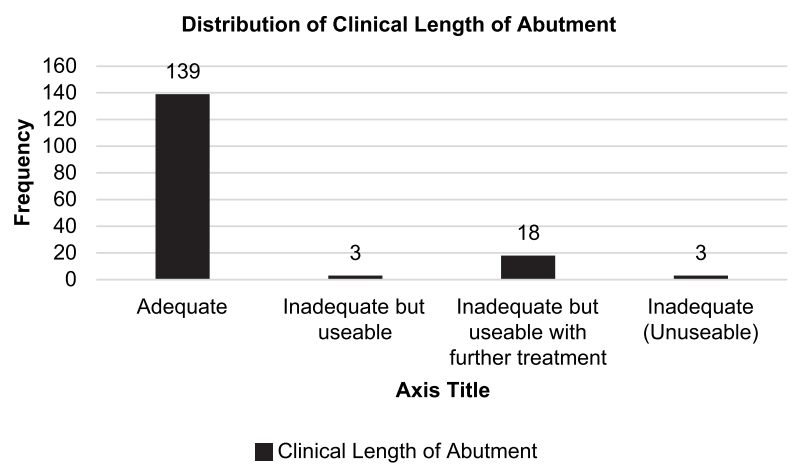

Figure-2. Distribution of clinical crown lengths of abutments.
Clinical crown length of abutments was compromised in $14.7 \%$ of the abutments, out of which $1.8 \%$ were usable without further treatment, $11.0 \%$ required further treatment to be used as abutment, while $1.8 \%$ were sufficiently compromised to preclude their use for FPD support (Figure-2). Clinical inclination of crown of abutment was compromised in $28.8 \%$ of the abutments, out of which $10.4 \%$ were usable without further treatment, $11.7 \%$ required further treatment to be used as abutment, while $6.7 \%$ had sufficient compromise to preclude their use for FPD support. Caries or fracture of abutment was observed in $22.1 \%$ of the abutments, out of which $4.3 \%$ were usable without further treatment, $15.3 \%$ required further treatment to be used as abutment, while $2.5 \%$ had sufficient compromise to preclude their use for FPD support.

No restoration was observed in $53.4 \%$ of the abutments while $47.9 \%$ of the abutments were restored. Of these, $26.4 \%$ had amalgam restorations, $13.5 \%$, had composite restorations and $8.0 \%$ showed glass ionomer cement restorations. No abutment showed metal or ceramic inlay or cast metal build-up (Figure-3). Compromised restoration quality was observed in $47.2 \%$ of the abutments, out of which $28.8 \%$ were usable without further treatment, $16.6 \%$ required further treatment to be used as abutment, while $0.6 \%$ had sufficient compromise to preclude their use for FDP support. On basis of the degree of compromise, $41.1 \%$ of the abutments were usable without further treatment or required a simple corrective procedure, $45.4 \%$ required a relatively complex procedure to make them useable while $11 \%$ had sufficient compromise to preclude their use for FPD support. Only $2.5 \%$ of abutments were classified as adequate (Figure-4).

\section{DISCUSSION}

The present study will help the dental practitioners to investigate the compromised conditions and their severity levels of the abutments and thus provide an idea on the conditions that need to be given special attention while examining the abutments. 


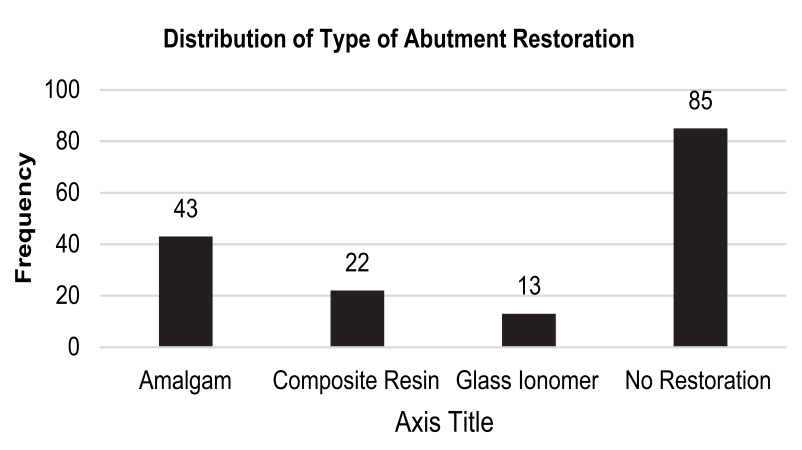

Abutment Restoration

Figure-3. Distribution of type of abutment restoration.

Restoration of missing teeth with fixed dental prosthesis is a treatment modality in routine dental practice owing high incidence of tooth loss secondary to caries and periodontal disease. ${ }^{12}$ Being an expensive treatment modality, involving extended chair-side time and laboratory procedure, fixed prosthodontic treatment should be offered to suitable patients only. ${ }^{13}$ In a metaanalysis conducted by Scurria et al. to formulate annual probability estimates for fixed partial denture survival showed a survival rate of $87 \%$ and $69 \%$ at 10 and 15 years respectively while Creugers et al estimated survival probabilities as $90 \%$ and $75 \%$ at 10 and 15 years, respectively. Focusing on abutment survival alone, 10 years' survival rate was estimated to be $96 \% .^{14,15}$

In a study by Ericson conducted over a period of three years, $3 \%$ of the studied population showed loss of abutment while a systematic review by Pjetursson et al. shows loss of abutment in 3.3\% of the population as a cause of FPD failure. ${ }^{16,17}$ Pre-treatment condition of the teeth to be employed as abutment for fixed prostheses is of crucial significance to the long term prognosis, very limited amount of literature focuses on the pre-treatment condition of natural teeth and their suitability to serve as abutment for fixed prostheses. $^{2,18}$

The present study was designed to determine the frequencies of common compromised coronal conditions of teeth that have been selected to serve as abutment for fixed dental prosthesis. The gender analysis of the study sample shows

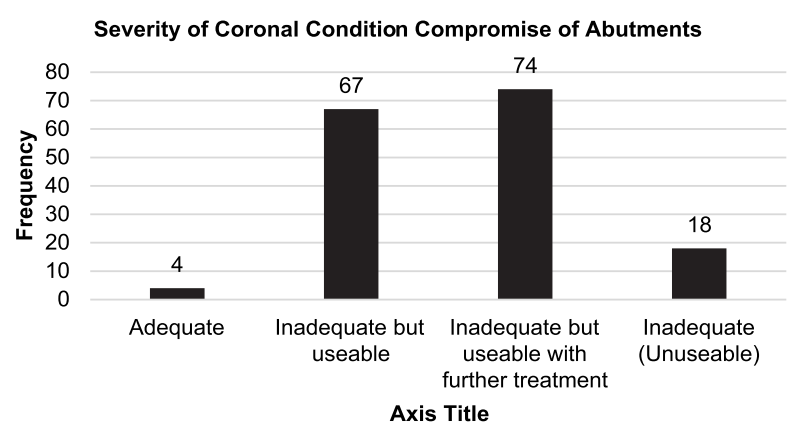

Severity of Coronal Condition Compromise of Abutments

Figure-4. Distribution of severity of coronal condition compromise of abutments.

a male dominance in patients seeking fixed prosthodontic treatment with a male to female ratio of 1.9:1. This is in agreement with a local study on metal ceramic fixed partial dentures which showed a male predominance in seeking fixed prosthodontic treatment (male to female ratio of 1.3:1). ${ }^{19}$ However, similar studies show a female dominance with percentages ranging from $62 \%$ in a study by Backer to $70 \%$ in a study by Kanno $\mathrm{T}$ et al. ${ }^{20,21}$ This difference may be attributed to different social circumstance, female literacy and awareness levels.

Maximum number of edentulous spaces in the present study population was located in mandibular posterior region while the least number of edentulous spaces were located in the mandibular anterior region. The same was true for a similar study by Memon et al. ${ }^{19}$ The unusual coronal morphology of the abutment teeth, was evaluated in terms of clinical length and inclination of the abutment. The clinical coronal length was compromised in $14.6 \%$ of the abutments. The presence of adequate amount tooth structure is critical for the long-term success of the abutment tooth. About $28 \%$ of the abutments considered in the present study displayed varying degree of abnormal inclination of the crown. Excessive clinical inclination may become an important prognostic factor for the subsequent fixed prostheses since it may require excessive reduction of the tooth structure. This may place pulpal health in jeopardy requiring endodontic treatment either prior to final cementation of the restoration or in later period of prosthesis 
service. ${ }^{22}$

The structural integrity of the abutments was assessed in terms of caries or fracture of the abutment. About $22 \%$ of the abutments showed structural compromise with about $3 \%$ of the abutments being compromised to an extent that they could not be employed for supporting the fixed prosthesis. This is in close agreement with values presented in 20-year retrospective survival study by Backer et al were $22.2 \%$ of the fixed prosthesis failed a result of carious lesion. ${ }^{20}$ Another study by Goodacre et al showed caries as a cause of complication in 18\% of FPD cases. This high incidence of carious lesions in abutment teeth carries important implications in long-term survival and prognosis of the FPD and demands institution of proper caries control and elimination procedures before fixed prosthodontic treatment is embarked upon to prevent development of advanced carious lesion/ decay of the abutments. ${ }^{23}$

The present study also evaluated the restorative status of the abutment teeth. About $47 \%$ of the abutments were restored. Amalgam was the most commonly used restorative material (26\%). This is because amalgam is a time tested restorative material which has been preferred over other restorative materials because of its ease of use, higher strength and minimal dissolution in oral environment. Composite resin was found in $12 \%$ of the abutments. Although composite shares many properties with amalgam but amalgam offers clear advantage in terms of rate of microleakage and strength. It is important to note that none of abutment had an indirect restoration like inlay or cast core. ${ }^{24}$

The present study showed that a very high percentage of abutments $(86.5 \%)$ required some sort of restorative treatment (simple or complex) to be able to function as abutment for FDP. This does not have a direct impact on the long term prognosis of the prosthesis. It also reflects high cost of treatment that will be incurred in order to make these abutments suitable for receiving fixed dental prosthesis.

\section{LIMITATIONS}

No objective criteria exist that could help in predicting the prognosis of the planned prosthesis with reference to the pre-treatment condition of the abutment teeth. More longitudinal studies need to be planned to evaluate 10 -15-year survival rate of fixed dental prosthesis based on the degree of compromise in coronal condition of the abutments.

\section{CONCLUSION}

Within the limitations of this study it can be concluded that about half of the studied abutments in subjects reporting for replacement of missing teeth with FDP were found to be compromised and a substantial proportion required complex restorative treatment/re-treatment prior to be used as FDP abutments. Hence proper examination and evaluation of entire abutment generally and restorative status specifically are of vital importance. Failure of restoration will ultimately result in failure of FDP. The results also show that about $11 \%$ of the abutments were unsuitable to be used as abutments. Hence FDP should be provided only after thorough evaluation in order to enhance the prognosis of prosthesis.

\section{Copyright $@ 07$ Dec, 2020.}

\section{REFERENCES}

1. Raj DA, Chander NG, Reddy JR, Balasubramaniam M. Clinical acceptability of PEEK fixed dental prosthesis in partially edentulous patient - A one year single arm pilot study. J Oral Biol Craniofac Res. 2020 OctDec; 10(4):523-528.

2. Ikai H, Kanno T, Kimura K. A review of clinical followup studies focusing on pre-treatment conditions of abutment and clinical examination parameters. Nihon Hotetessu Shaika Gakkai Zasshi. 2006; 50:24555.

3. Moeen F. Effects of varying height and taper on the fatigue performance of full coverage Tescera ATL Ceromer Crowns. J Pak Dent Assoc. 2005; 14:163-70.

4. Al Refai R, Saker S. Clinical and radiographic assessment of reasons for replacement of metalceramic fixed dental prostheses in patients referring to dental school. J Clin Exp Dent. 2018 Jan 1; 10(1):7580. 
6. Saleem T, Amjad F, Bhatti MU. Complications associated with tooth supported fixed dental prostheses amongst patients visiting University College of Dentistry Lahore. Pak Oral Dent J. 2013; 33:207-11.

7. Celikoglu M, Miloglu O, Kazanci F. Frequency of agenesis, impaction, angulation and related pathologic changes of third molar teeth in orthodontic patients. J Oral Maxillofac Surg. 2010; 68:990-5.

8. Demirci M, Tuncer S, Yuceokur AA, Davis MM, Hilton $\mathrm{TJ}$, Benson S, et al. Unmet dental needs in rural primary care: A clinic-community, and practicebased research network collaborative. J Am Board Fam Med. 2010; 23:514-22.

9. Demirci M, Tuncer S, Yuceokur AA. Prevalence of caries on individual tooth surfaces and its distribution by age and gender in university clinic patients. Eur $\mathrm{J}$ Dent. 2010; 4:270-9.

10. Iqbal MK, Kim S. For teeth requiring endodontic treatment, what are the differences in outcomes of restored endodontically treated teeth compared to implant-supported restorations? Int J Oral Maxillofac Implants. 2007; 22 suppl:96-116.

11. Balevi B. Root canal therapy, fixed partial dentures and implant-supported crowns, have similar shortterm survival rates. Evid Based Dent. 2008; 9:15-7.

12. Bernardo M, Martin MD, Leroux BG, DeRouen TA. Survival and reasons for failure of amalgam versus composite posterior restorations placed in a randomized clinical trial. J Am Dent Assoc. 2007; 138:775-83.

13. Sonbul $H$, Birkhed $D$. Risk profile and quality of dental restorations: A cross-sectional study. Acta Odontol Scand. 2010; 68:122-8.

14. Klineberg I. Ideas about the future of prosthodontics. Int J Prosthodont 2000; 13:81-82.

15. Kharat SS, Tatikonda A, Raina S, Gubrellay P, Gupta $\mathrm{N}$, Asopa SJ. In vitro evaluation of the accuracy of seating cast metal fixed partial denture on the abutment teeth with varying degree of convergence angle. Journal of clinical and diagnostic research. 2015; 9(7):56-60.
16. Scurria MS, Bader JD, Shugars DA. Meta-analysis of fixed partial denture survival: Prostheses and abutments. J Prosthet Dent. 1998; 79:459-64.

17. Creugers $\mathrm{NH}$, Käyser $\mathrm{AF}$, van't Hof MA. A meta-analysis of durability data on conventional fixed bridges. Community Dent Oral Epidemiol. 1994; 22:448-52.

18. Ericson G, Nilson H, Bergman B. Cross-sectional study of patients fitted with fixed partial dentures with special reference to the caries situation. Scand J Dent Res. 1990; 98:8-16.

19. Tan K, Pjetursson BE, Lang NP, Chan ES. A systematic review of the survival and complication rates of fixed partial dentures (FPDs) after an observation period of at least 5 years. Clin Oral Implants Res. 2004; 15:654-66.

20. Libby G, Arcuri MR, LaVelle WE, Hebl L. Longevity of fixed partial dentures. J Prosthet Dent. 1997; 78:12731.

21. Ghani F. Memon MR. Complications in Metal-Ceramic Fixed Dental Prostheses among patients reporting to a Teaching Dental Hospital. JLUMHS. 2010; 9:17-22.

22. De Backer H, Van Maele G, De Moor N, Van den Berghe L, De Boever J. A 20-year retrospective survival study of fixed. Int J Prosthodont. 2006; 19:143-53.

23. Ikai H, Kanno T, Kimura K, Sasaki K. A retrospective study of fixed dental prostheses without regular maintenance. J Prosthodont Res. 2010; 54:173-8.

24. Rosential SF, Land M, Fujimoto J. Principals of tooth preparation. In: Contemporary fixed prosthodontics. 4th ed. St. Louis: Elsevier; 2006. p. 209-51.

25. Goodacre CJ, Bernal G, Rungcharassaeng K, Kan JY. Clinical complications in fixed prosthodontics. J Prosthet Dent. 2003; 90:31-41. 


\begin{tabular}{|c|c|c|c|}
\hline \multicolumn{4}{|c|}{ AUTHORSHIP AND CONTRIBUTION DECLARATION } \\
\hline Sr. \# & Author(s) Full Name & Contribution to the paper & Author(s) Signature \\
\hline 1 & Aamna Khalil & $\begin{array}{l}\text { Original idea of research, Statistics } \\
\& \text { Literature review. }\end{array}$ & \\
\hline 2 & Zarah Afreen & $\begin{array}{l}\text { Data collection, Statistics, } \\
\text { Literature review. }\end{array}$ & \\
\hline 3 & Ammarah Afreen & Data collection, Literature review. & $-A x$ \\
\hline 4 & Umer Javed & Statistics, Literature review. & \\
\hline 5 & Shoaib Rahim & Literature review. & $\Leftrightarrow$ \\
\hline 6 & Adil Asim & Literature review. & 争 \\
\hline
\end{tabular}

